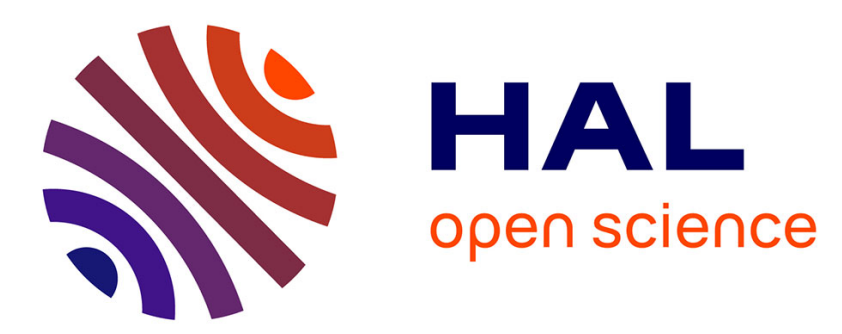

\title{
Second-order lattice Fokker-Planck algorithm from the trapezoidal rule
}

Benjamin Rotenberg, Daniele Moroni

\section{To cite this version:}

Benjamin Rotenberg, Daniele Moroni. Second-order lattice Fokker-Planck algorithm from the trapezoidal rule. Physical Review E: Statistical, Nonlinear, and Soft Matter Physics, 2006, 74 (3), pp.037701. 10.1103/PhysRevE.74.037701 . hal-01897053

\section{HAL Id: hal-01897053 https://hal.sorbonne-universite.fr/hal-01897053}

Submitted on 16 Oct 2018

HAL is a multi-disciplinary open access archive for the deposit and dissemination of scientific research documents, whether they are published or not. The documents may come from teaching and research institutions in France or abroad, or from public or private research centers.
L'archive ouverte pluridisciplinaire HAL, est destinée au dépôt et à la diffusion de documents scientifiques de niveau recherche, publiés ou non, émanant des établissements d'enseignement et de recherche français ou étrangers, des laboratoires publics ou privés. 


\title{
Second-order lattice Fokker-Planck algorithm from the trapezoidal rule
}

\author{
Benjamin Rotenberg ${ }^{1,2}$ and Daniele Moroni ${ }^{3}$ \\ ${ }^{1}$ Université Pierre et Marie Curie-Paris6, Laboratoire Liquides Ioniques et Interfaces Chargées, UMR CNRS 7612, 4 pl. Jussieu, Paris F- \\ 75005 \\ ${ }^{2}$ ANDRA, Parc de la Croix Blanche, $1 / 7$ rue Jean Monnet 92298 Châtenay Malabry, cedex F-75005, France \\ ${ }^{3}$ Department of Chemistry, University of Cambridge, Lensfield Road, Cambridge CB2 1EW, United Kingdom
}

(Received 26 April 2006; published 5 September 2006)

\begin{abstract}
The well-established procedure of deriving lattice Boltzmann schemes using the trapezoidal rule for time integration is generalized to the recently introduced lattice Fokker-Planck method.
\end{abstract}

DOI: 10.1103/PhysRevE.74.037701

PACS number(s): 47.11.-j, 47.10.-g, 05.20.Dd

In a series of recent papers [1-4] a lattice method to solve the Fokker-Planck (FP) kinetic equation has been introduced. The equation models a two-component system, e.g., solute and solvent, when a separation of time scales is expected. One can then adopt an effective one-component description and consider only the solute distribution function while the FP collision operator takes into account the effect of the solvent. The resulting kinetic equation can be solved numerically within the framework of the Lattice-Boltzmann (LB) method [5-8]. The method is usually applied to kinetic equations of the Bhatnagar-Gross-Krook (BGK) type to reproduce hydrodynamic behavior, but can also be used to construct lattice algorithms to solve more general types of equations [5].

The derivation of the lattice FP (LFP) algorithm, described in detail in [1], follows a systematic procedure [9-12] involving two separate steps: discretization in real and velocity space on the one hand, discretization in time on the other hand. In the first step the positions $\mathbf{x}$ are considered points on a lattice, and the velocities can only assume a finite set of values $\mathbf{v}_{i}, i=1, \ldots, b$ corresponding to lattice links. The resulting continuous-time equation for the lattice distribution functions $g_{i}$ is

$$
\partial_{t} g_{i}(\mathbf{x} ; t)+v_{i \alpha} \partial_{\alpha} g_{i}(\mathbf{x} ; t)=\hat{L}_{i}[g(\mathbf{x} ; t)],
$$

where $v_{i \alpha}$ are Cartesian components in generic dimension $D$, Greek indices run from 1 to $D, \partial_{t}=\partial / \partial t$ and $\partial_{\alpha}=\partial / \partial x_{\alpha}$ are, respectively, time and space derivatives, and summation convention is assumed. On the right-hand side appears the $i$ th component $\hat{L}_{i}$ of the lattice collision operator $\hat{L}$ acting on the populations $g_{i}(\mathbf{x} ; t)$ denoted by the collective symbol $g$. The lattice operator is derived from the continuous FP operator using a Gauss-Hermite quadrature and involves the moments of the $g_{i}$ in velocity space, namely the density $\rho=\Sigma_{i} g_{i}$, the current $J_{\alpha}=\sum_{i} g_{i} v_{i \alpha}$, and the stress tensor $P_{\alpha \beta}=\sum_{i} g_{i} v_{i \alpha} v_{i \beta}$. The second step is achieved by integrating (1) between $t$ and $t+\Delta t$. Let $g(s)$ denote the time-shifted populations $g_{i}\left(\mathbf{x}+\mathbf{v}_{i} s ; t+s\right)$. Then

$$
g^{\prime}-g=\int_{0}^{\Delta t} \hat{L}[g(s)] d s,
$$

where $g^{\prime}=g(\Delta t)$ are the populations $g_{i}\left(\mathbf{x}+\mathbf{v}_{i} \Delta t ; t+\Delta t\right)$. Approximation of the integral by the rectangle rule results in a numerical scheme which is only first-order accurate in $\Delta t$. In [1] the Chapman-Enskog analysis of the algorithm allowed us to evaluate the $O\left(\Delta t^{2}\right)$ corrections analytically, so that it was possible to derive a heuristic second-order scheme, referred to as "corrected scheme." In this paper, we show that it is possible to achieve second-order accuracy of the LFP scheme by extending an idea commonly used in LB methods, the approximation of the integral in (2) by the trapezoidal rule [13-16].

The trapezoidal rule is an average of the starting and final points of the integration interval

$$
\int_{0}^{\Delta t} \hat{L}[g(s)] d s=\frac{\Delta t}{2}\left(\hat{L}\left[g^{\prime}\right]+\hat{L}[g]\right)+O\left(\Delta t^{3}\right)
$$

It is similar in spirit to the Crank-Nicholson method for ordinary differential equations and has second-order accuracy [17]. The presence of the collision operator at time $t+\Delta t$ makes the combination of (2) and (3) unusable as such. However, following [13-16] we introduce the mapping

$$
\tilde{g}=g-\frac{\Delta t}{2} \hat{L}[g]
$$

and rewrite (3) as

$$
\widetilde{g}^{\prime}-\widetilde{g}=\Delta t \hat{L}[g]+O\left(\Delta t^{3}\right),
$$

where the right-hand side now depends on the populations $g_{i}$ at time $t$ only. Equation (5) can be turned into an operational form by expressing the collision operator in terms of the shifted populations (4).

It is instructive to recall the final form of the scheme in the case of the BGK collision operator

$$
\hat{L}^{B G K}[g]=-\tau^{-1}\left(g-g^{e q}\right) \doteq-\tau^{-1} L^{B G K}[g],
$$

where $\tau^{-1}$ is the collision frequency, $g^{e q}$ is the discretized Maxwell-Boltzmann equilibrium population, depending only on the moments $\rho, \mathbf{J}$, and we have introduced the operator $L^{B G K}[g]=g-g^{e q}$. Using the definition (4), Eq. (5) is rewritten as

$$
\tilde{g}^{\prime}-\tilde{g}=-\frac{\tau^{-1} \Delta t}{1+\tau^{-1} \Delta t / 2} L^{B G K}[\tilde{g}]
$$

and, in particular, we can compute $\widetilde{g}^{e q}$ because the zeroth and first moments of $g$ and $\widetilde{g}$ coincide. This is not true for the 
second moment tensor $\mathcal{P}$, and indeed the trapezoidally evolving distributions $g$ must be used for the sampling. In practice one does not invert (4) (which is not trivial) but just uses the associated moment relations.

In the LFP case $\tau^{-1}$ is replaced by the friction $\gamma$ and $L^{B G K}$ by $[1]$

$$
L_{i}[g] / w_{i}=\left[J_{\alpha}-J_{\alpha}^{e q}\right] \frac{\mathcal{H}_{i, \alpha}^{(1)}}{v_{T}^{2}}+2\left[P_{\alpha \beta}-P_{\alpha \beta}^{e q}\right] \frac{\mathcal{H}_{i, \alpha \beta}^{(2)}}{2 v_{T}^{4}},
$$

where the Hermite polynomials [18] are $\mathcal{H}_{i, \alpha}^{(1)}=v_{i \alpha}, \mathcal{H}_{i, \alpha \beta}^{(2)}$ $=v_{i \alpha} v_{i \beta}-v_{T}^{2} \delta_{\alpha \beta}, w_{i}$ are appropriate weights, $v_{T}$ is the thermal velocity,

$$
\begin{gathered}
J_{\alpha}^{e q} \doteq u_{\alpha}^{E} \rho \\
P_{\alpha \beta}^{e q} \doteq v_{T}^{2} \rho \delta_{\alpha \beta}+\left(u_{\alpha}^{E} J_{\beta}+u_{\beta}^{E} J_{\alpha}\right) / 2
\end{gathered}
$$

and the external velocity $u_{\alpha}^{E}$ is defined such that the constant external field $m \gamma u_{\alpha}^{E}$ acts on the solute particles of mass $m$. The collision operator involves now up to the second moment, and computing the moments on both sides of (4) we get

$$
\begin{gathered}
\tilde{\rho}=\rho, \\
\tilde{J}_{\alpha}=\left(1+\frac{\gamma \Delta t}{2}\right) J_{\alpha}-\frac{\gamma \Delta t}{2} J_{\alpha}^{e q}, \\
\tilde{P}_{\alpha \beta}=(1+\gamma \Delta t) P_{\alpha \beta}-\gamma \Delta t P_{\alpha \beta}^{e q},
\end{gathered}
$$

showing that only the zeroth moments of $g$ and $\widetilde{g}$ coincide. The operational form of the trapezoidal LFP algorithm is specified by

$$
\widetilde{g}^{\prime}-\widetilde{g}=-\gamma \Delta t \widetilde{L}[\widetilde{g}],
$$

where the operator $\widetilde{L}[\tilde{g}]$ must satisfy $\tilde{L}[\tilde{g}] \equiv L[g]$. In the BGK case $\tilde{L}[\tilde{g}]$ is just $L[\tilde{g}] /\left(1+\tau^{-1} \Delta t / 2\right)$, see Eq. (7). In the LFP case instead we have to invert Eqs. (11). The collision operator $\tilde{L}$ can be computed explicitly, but in a computer implementation one can follow a recursive procedure. The zeroth moments coincide, and the first becomes

$$
J_{\alpha}=\frac{\widetilde{J}_{\alpha}+(\gamma \Delta t / 2) J_{\alpha}^{e q}}{1+\gamma \Delta t / 2}
$$

where $J_{\alpha}^{e q}$ can be computed because it contains only $\rho=\tilde{\rho}$. The second moment is then

$$
P_{\alpha \beta}=\frac{\widetilde{P}_{\alpha \beta}+\gamma \Delta t P_{\alpha \beta}^{e q}}{1+\gamma \Delta t}
$$

and again $P_{\alpha \beta}^{e q}$ contains only $\rho$ and $J_{\alpha}$ which are now available. Substituting relations (13a) and (13b) into (8) we get the desired operator

$$
\tilde{L}_{i}[\widetilde{g}] / w_{i}=\frac{\widetilde{J}_{\alpha}-J_{\alpha}^{e q}}{1+\gamma \Delta t / 2} \frac{\mathcal{H}_{i, \alpha}^{(1)}}{v_{T}^{2}}+2 \frac{\widetilde{P}_{\alpha \beta}-P_{\alpha \beta}^{e q}}{1+\gamma \Delta t} \frac{\mathcal{H}_{i, \alpha \beta}^{(2)}}{2 v_{T}^{4}} .
$$

Equations (11) are specific to the FP case, but the recursive procedure only exploits the fact that the collision matrix is lower triangular [1] and has hence wider applicability.

The trapezoidal algorithm can be summarized as follows. Choose the friction $\gamma$, which at variance with the scheme of Ref. [1] does not need any rescaling. The evolution is performed on $\widetilde{g}$, while initial conditions and sampling use the trapezoidally corrected bare moments $\rho, \mathbf{J}, \mathcal{P}$, which are the true hydrodynamic variables. Then given $\widetilde{g}$ at time $t$ : (1) Compute the tilde moments $\widetilde{\rho}, \widetilde{J}_{\alpha}, \widetilde{P}_{\alpha \beta}$ and hence the bare equilibrium moments $J_{\alpha}^{e q}, P_{\alpha \beta}^{e q}$ using the recursive procedure; (2) Compute the post-collision populations $\widetilde{L}[\widetilde{g}]$ using (14); (3) Stream $\widetilde{g}$ to $\widetilde{g}^{\prime}$ at time $t+\Delta t$. The hydrodynamic variables are sampled by means of Eqs. (13a) and (13b). The computational effort is comparable to that of previously proposed schemes because the new collision operator just requires the inversion equations (13a) and (13b) which only involve a few more floating point operations. In particular, the linear scaling with lattice size is preserved.

It can be shown that the heuristic scheme introduced in [1] can be recovered by (a) using the tilde variables in place of the bare ones [also in the definition of the equilibrium moments (9) and (10)] and an effective friction $\tilde{\gamma}=\gamma /(1+\gamma \Delta t / 2)$, (b) neglecting a $O\left(\Delta t^{2}\right)$ term in the lattice collision operator (14), and (c) expanding to order $\Delta t$ Eqs. (13a) and (13b) for the sampled moments (which were denoted with stars in [1]). This explains why the heuristic scheme has a larger error of order $\Delta t^{2}$ than the trapezoidal algorithm (see below), and also shows that the evolution of the density and the current are identical in both schemes.

The present algorithm propagates the populations from $t$ to $t+\Delta t$ with second-order accuracy. However, the error after many time steps could be worse. We now show that this order of accuracy is preserved for the long-time behavior by performing a Chapman-Enskog analysis. The populations $g_{i}$ and the time and space derivatives are expanded in powers of a small parameter $\epsilon$ analogous to the Knudsen number used in the LB case:

$$
\begin{gathered}
g_{i}=g_{i}^{(0)}+\epsilon g_{i}^{(1)}+\epsilon^{2} g_{i}^{(2)} \\
\partial_{t}=\epsilon \partial_{t}^{(1)}+\epsilon^{2} \partial_{t}^{(2)}, \quad \partial_{\alpha}=\epsilon \partial_{\alpha}^{(1)} .
\end{gathered}
$$

In the FP case however, the relevant expansion parameter is rather defined as $\epsilon=\left(v_{T} / R\right) / \gamma$, where $R$ is the solute radius. The small $\epsilon$ expansion is thus a high friction limit, where a large number of solute-solvent collisions occur while the solute moves over its own radius [19]. All hydrodynamic moments, which are linear combinations of the $g_{i}$ 's, are expanded as in Eq. (15a). We can approximate the populations $\widetilde{g}_{i}^{\prime} \equiv \widetilde{g}_{i}\left(\mathbf{x}+\mathbf{v}_{i} \Delta t ; t+\Delta t\right)$ by a Taylor expansion around $\tilde{g}_{i}$ $\equiv \widetilde{g}_{i}(\mathbf{x} ; t)$, and write up to second order $\widetilde{g}_{i}^{\prime}-\widetilde{g}_{i}=\Delta t \hat{C}_{S}\left[\widetilde{g}_{i}\right]$, where 


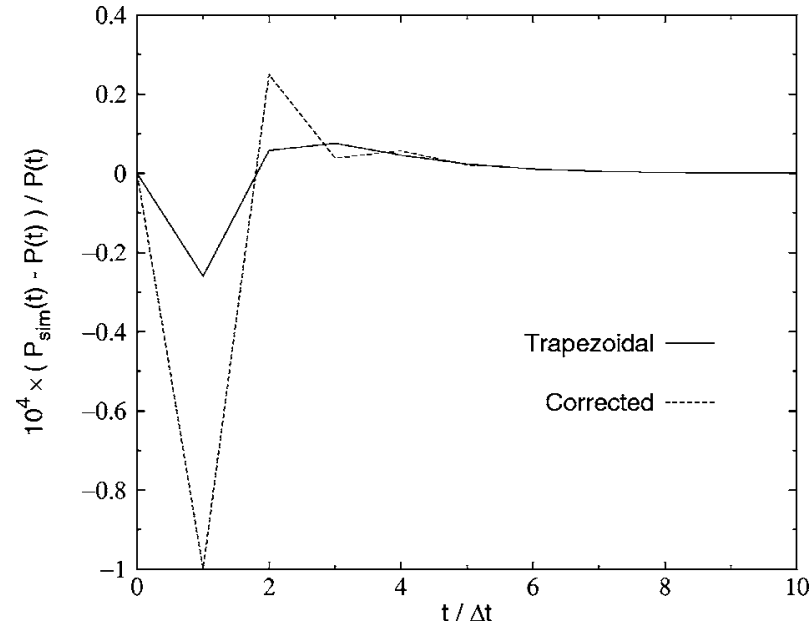

FIG. 1. Relative error on the stress $P$ as a function of time for a constant, uniform applied field on an infinite homogeneous system at a friction $\gamma \Delta t=0.9$. The trapezoidal scheme (solid line) and the corrected scheme of Ref. [1] (dashed lined), are compared to the analytical solution Eq. (24). The trapezoidal algorithm shows better agreement with theory.

$$
\hat{C}_{S}\left[\widetilde{g}_{i}\right]=\left[\partial_{t}+v_{i \alpha} \partial_{\alpha}+\Delta t\left(\partial_{t}+v_{i \alpha} \partial_{\alpha}\right)^{2} / 2\right] \widetilde{g}_{i} .
$$

Using Eqs. (15) the operator $\hat{C}_{S}$ is expanded as

$$
\begin{aligned}
\hat{C}_{S}\left[\widetilde{g}_{i}\right]= & \epsilon\left\{\partial_{t}^{(1)} \widetilde{g}_{i}^{(0)}+\partial_{\alpha}^{(1)} v_{i \alpha} \widetilde{g}_{i}^{(0)}\right\}+\epsilon^{2}\left\{\partial_{t}^{(1)} \widetilde{g}_{i}^{(1)}+\partial_{\alpha}^{(1)} v_{i \alpha} \widetilde{g}_{i}^{(1)}\right. \\
& +\partial_{t}^{(2)} \widetilde{g}_{i}^{(0)}+\left[\partial_{t}^{(1)}\left(\partial_{t}^{(1)} \widetilde{g}_{i}^{(0)}+\partial_{\beta}^{(1)} v_{i \beta} \widetilde{g}_{i}^{(0)}\right)\right. \\
& \left.\left.+\partial_{\alpha}^{(1)}\left(\partial_{t}^{(1)} v_{i \alpha} \widetilde{g}_{i}^{(0)}+\partial_{\beta}^{(1)} v_{i \alpha} v_{i \beta} \widetilde{g}_{i}^{(0)}\right)\right] \Delta t / 2\right\} .
\end{aligned}
$$

As for the lattice collision operator (8), the expansion acts order by order on the moments and we can write $L_{i}[g]=L_{i}^{(0)}+\epsilon L_{i}^{(1)}+\epsilon^{2} L_{i}^{(2)}$ where

$$
L_{i}^{(\mu)} / w_{i}=\left[J_{\alpha}^{(\mu)}-J_{\alpha}^{e q,(\mu)}\right] \frac{\mathcal{H}_{i, \alpha}^{(1)}}{v_{T}^{2}}+2\left[P_{\alpha \beta}^{(\mu)}-P_{\alpha \beta}^{e q,(\mu)}\right] \frac{\mathcal{H}_{i, \alpha \beta}^{(2)}}{2 v_{T}^{4}}
$$

for $\mu=0,1,2$. After equating terms of the same order in $\epsilon$ in Eqs. (17) and (18), the moment equations can be computed at each order. For the zeroth moment equation one can just sum both sides of the equations over $i$, for the next moments one must first multiply by $v_{i \gamma}, v_{i \gamma} v_{i \delta}$, and so on. For each order in $\epsilon$, we can compute different moment equations. The computations are carried out using the orthonormality relations of Hermite polynomials, see, e.g., $[1,18]$. To order 0 , the zeroth moment does not give any information, while the first and second moment read

$$
\begin{gathered}
0=-\gamma\left[J_{\gamma}^{(0)}-J_{\gamma}^{e q,(0)}\right], \\
0=-2 \gamma\left[P_{\gamma \delta}^{(0)}-P_{\gamma \delta}^{e q,(0)}\right] .
\end{gathered}
$$

At this stage it is important to note that using (19) and (11) one also finds

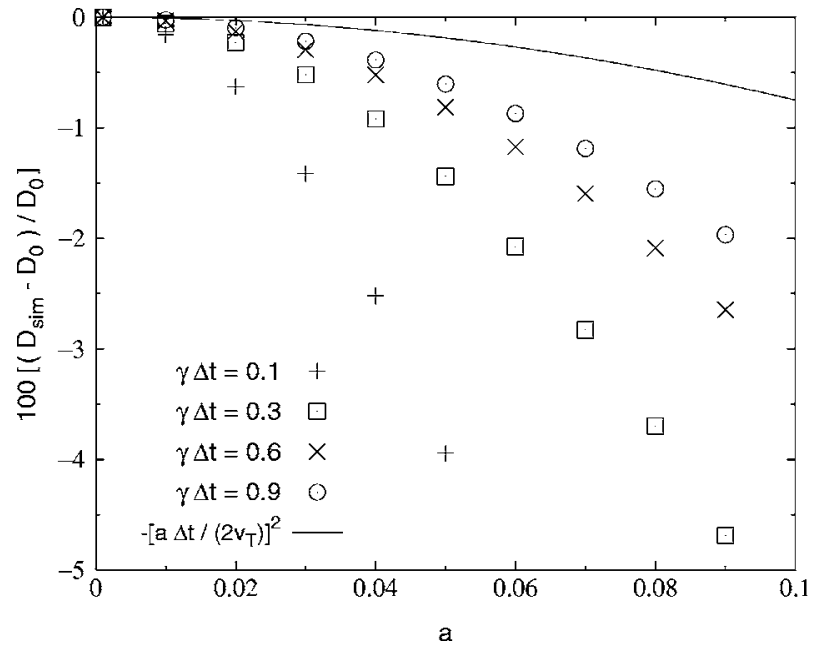

FIG. 2. Relative error for the diffusion coefficient. In the trapezoidal scheme the error is almost equal to $-\left[a \Delta t /\left(2 v_{T}\right)\right]^{2}$ (solid line), independently of the friction $\gamma$, while the error is larger in the corrected scheme (symbols) and does depend on the friction. Acceleration $a$ is in units $\Delta x / \Delta t^{2}$.

$$
\tilde{\rho}^{(\mu)}=\rho^{(\mu)} \forall \mu, \quad \widetilde{J}_{\gamma}^{(0)}=J_{\gamma}^{(0)}, \quad \tilde{P}_{\gamma \delta}^{(0)}=P_{\gamma \delta}^{(0)} .
$$

To order $\epsilon$ we find for the zeroth, first, and second moments

$$
\begin{gathered}
\partial_{t}^{(1)} \widetilde{\rho}^{(0)}+\partial_{\alpha}^{(1)} \widetilde{J}_{\alpha}^{(0)}=0, \\
\partial_{t}^{(1)} \widetilde{J}_{\gamma}^{(0)}+\partial_{\alpha}^{(1)} \widetilde{P}_{\alpha \gamma}^{(0)}=-\gamma\left[J_{\gamma}^{(1)}-J_{\gamma}^{e q,(1)}\right], \\
\partial_{t}^{(1)} \widetilde{P}_{\gamma \delta}^{(0)}+\partial_{\alpha}^{(1)} \widetilde{Q}_{\alpha \gamma \delta}^{(0)}=-2 \gamma\left[P_{\gamma \delta}^{(1)}-P_{\gamma \delta}^{e q,(1)}\right] .
\end{gathered}
$$

For the order $\epsilon^{2}$ we use the results of Eq. (21), and we only consider the zeroth and first moment equations. Using Eqs. (21) and (11) we get after some algebra

$$
\begin{gathered}
\partial_{t}^{(2)} \rho^{(0)}+\partial_{t}^{(1)} \rho^{(1)}+\partial_{\alpha}^{(1)} J_{\alpha}^{(1)}=0, \\
\partial_{t}^{(2)} J_{\gamma}^{(0)}+\partial_{t}^{(1)} J_{\gamma}^{(1)}+\partial_{\alpha}^{(1)} P_{\alpha \gamma}^{(1)}=-\gamma\left[J_{\gamma}^{(2)}-J_{\gamma}^{e q,(2)}\right] .
\end{gathered}
$$

We now reconstruct the total time and space derivative by forming the combinations $\epsilon^{1}(21 a)+\epsilon^{2}(22 a)$ for the zeroth moment, and $\epsilon^{0}(19 a)+\epsilon^{1}(21 b)+\epsilon^{2}(22 b)$ for the first, where in (21a) and (21b) the moments can be replaced by their bare counterparts, according to (20). Recognizing that $\partial_{\alpha} X=\epsilon \partial_{\alpha}^{(1)}$ $\times\left(X^{(0)}+\epsilon X^{(1)}\right)=\epsilon \partial_{\alpha}^{(1)} X^{(0)}+\epsilon^{2} \partial_{\alpha}^{(1)} X^{(1)}$, where $X$ is any of the moments, and that $\partial_{t} X=\epsilon \partial_{t}^{(1)} X^{(0)}+\epsilon^{2} \partial_{t}^{(2)} X^{(0)}+\epsilon^{2} \partial_{t}^{(1)} X^{(1)}$, the combinations can be written as

$$
\begin{gathered}
\partial_{t} \rho+\partial_{\alpha} J_{\alpha}=0, \\
\partial_{t} J_{\gamma}+\partial_{\alpha} P_{\alpha \gamma}=-\gamma\left(J_{\gamma}-J_{\gamma}^{e q}\right),
\end{gathered}
$$

which coincide with the moment equations obtained from the continuous FP equation [1].

Stability analysis of LB schemes requires $-2<\lambda<0$, where $\lambda$ is any eigenvalue of the associated collision matrix [6]. In the present trapezoidal LFP case the eigenvalues 
can be computed along the lines of [1] and are $0,-\gamma \Delta t /(1$ $+\gamma \Delta t / 2)$, and $-2 \gamma \Delta t /(1+\gamma \Delta t)$. The stability inequalities are always satisfied and the scheme is unconditionally stable. This is an interesting effect that we also verified numerically. We note that this does not also mean that the scheme unconditionally reproduces Eqs. (23). The numerical criterions of small Mach number (weak external fields) and friction $(\gamma \Delta t<1)$ found in [1] continue to apply for a faithful reproduction of the macroscopic equations.

We now reconsider two numerical tests introduced in [1]. In the first example, a constant external field $m \gamma u^{E}$ is applied in a one-dimensional system with periodic boundary conditions, initially homogeneous at density $\rho_{0}$ and vanishing velocity. The current obeys $J(t)=\rho u^{E}[1-\exp (-\gamma t)]$ and the evolution equation for the second moment $\partial_{t} P=-2 \gamma\left(P-\rho v_{T}^{2}-u^{E} J\right)$ has the analytical solution

$$
\begin{aligned}
P(t)= & \rho\left[\left(u^{E}\right)^{2}+v_{T}^{2}\right]-2 \rho\left(u^{E}\right)^{2} e^{-\gamma t} \\
& +\left\{P(0)-2 \rho\left[-\left(u^{E}\right)^{2}+v_{T}^{2}\right]\right\} e^{-2 \gamma t}
\end{aligned}
$$

The system is initialized with a Maxwell-Boltzmann distribution at fixed macroscopic $\rho=\rho_{0}, J / \rho \equiv u=0$. The simulated moments $\widetilde{\rho}, \tilde{u}=\widetilde{J} / \widetilde{\rho}$ are set to fulfill these conditions, and in this case both the corrected and the trapezoidal scheme reduce to setting $\widetilde{\rho}=\rho_{0}$ and $\widetilde{u}=-u^{E} \gamma \Delta t / 2$. The quantity $\widetilde{P}(0)$ is completely determined and equal to $\rho\left(v_{T}^{2}+\widetilde{u}^{2}\right)$ which in turn gives $P(0)=\rho v_{T}^{2}+\rho \tilde{u}^{2} /(1+\gamma \Delta t)$ for the trapezoidal scheme and $P(0)=\rho v_{T}^{2}-\rho \widetilde{u}^{2}$ for the corrected scheme [in the continuous case $P(0)=\rho v_{T}^{2}$. Simulation results show that the current $J$ is exactly the same in both schemes and fails to correctly reproduce the continuous results for $\gamma \Delta t>1$, but the stress tensor $P$ is closer to the analytical result in the trapezoidal scheme (see Fig. 1).

In the second example we consider the same system with bounce-back no-slip reflecting boundary conditions [5]. Let $a=\gamma u^{E}$ denote the constant applied acceleration. Accumula- tion due to migration results in a concentration gradient which is the source of a diffusive flux opposed to the applied field. From the balance of fluxes, we find at equilibrium the barometric law for the density $\rho_{e q}(x) \propto \exp \left(a x / v_{T}^{2}\right)$ $\equiv \exp \left(u^{E} x / D_{0}\right)$, where we defined the diffusion coefficient $D_{0}=v_{T}^{2} / \gamma$ according to Einstein's relation. From an exponential fit of the data we derive a simulated diffusion coefficient $D_{\text {sim }}$ upon dividing $u^{E}$ by the measured slope. The relative error reported in Fig. 2 shows that the values of $D_{\text {sim }}$ are closer to $D_{0}$ than in the case of the corrected scheme. In the former case the error is almost equal to $-\left[a \Delta t /\left(2 v_{T}\right)\right]^{2}$, independently of the friction $\gamma$, and smaller than in the latter case, where in addition the error depends on the friction.

We have presented a new lattice Fokker-Planck algorithm, based on the trapezoidal rule for time integration. Generalizing a well-established procedure of LB methods we have derived a computational scheme for the integration of the LFP equation with second-order accuracy. The ChapmanEnskog analysis and numerical tests of this scheme confirm the increased accuracy with respect to the integration schemes of Refs. [1-3], where LFP was introduced. We thus recommend the use of the trapezoidal algorithm in the implementations of the LFP method. Further improvements are expected by using refined no-slip boundary conditions [20]. A LB scheme in the presence of nonconserved fields has recently been derived in [21]. Work is in progress on the generalization of the present method to cases where the external field is self-consistent or time dependent.

\section{ACKNOWLEDGMENTS}

Fruitful discussions with J.-P. Hansen, S. Melchionna, and S. Succi are gratefully acknowledged. B.R. acknowledges financial support from the Agence Nationale pour la Gestion des Déchets Radioactifs (ANDRA, France). D.M. acknowledges financial support from Schlumberger Cambridge Research.
[1] D. Moroni, B. Rotenberg, J.-P. Hansen, S. Succi, and S. Melchionna, Phys. Rev. E 73, 066707 (2006).

[2] S. Melchionna, S. Succi, and J.-P. Hansen, Int. J. Mod. Phys. C 17, 459 (2006).

[3] S. Melchionna, S. Succi, and J.-P. Hansen, Phys. Rev. E 73, 017701 (2006).

[4] B. Rotenberg et al., J. Chem. Phys. 124, 154701 (2006).

[5] S. Succi, The Lattice Boltzmann Equation for Fluid Dynamics and Beyond (Oxford University Press, Oxford, 2001).

[6] R. Benzi, S. Succi, and M. Vergassola, Phys. Rep. 222, 145 (1992).

[7] S. Chen and G. Doolen, Annu. Rev. Fluid Mech. 30, 329 (1998).

[8] S. Succi, I. V. Karlin, and H. Chen, Rev. Mod. Phys. 74, 1203 (2002).

[9] X. He and L.-S. Luo, Phys. Rev. E 55, R6333 (1997).

[10] X. He and L.-S. Luo, Phys. Rev. E 56, 6811 (1997).

[11] X. Shan and X. He, Phys. Rev. Lett. 80, 65 (1998).
[12] N. S. Martys, X. Shan, and H. Chen, Phys. Rev. E 58, 6855 (1998).

[13] X. He, X. Shan, and G. D. Doolen, Phys. Rev. E 57, R13 (1998).

[14] X. He, S. Chen, and G. D. Doolen, J. Comput. Phys. 146, 282 (1998).

[15] P. J. Dellar, Phys. Rev. E 64, 031203 (2001).

[16] P. J. Dellar, J. Comput. Phys. 190, 351 (2003).

[17] M. Abramowitz and I. A. Stegun, Handbook of Mathematical Functions with Formulas, Graphs, and Mathematical Tables, 9th printing (Dover, New York, 1972).

[18] H. Grad, Commun. Pure Appl. Math. 2, 325 (1949).

[19] L. Bocquet, Am. J. Phys. 65, 140 (1997).

[20] M. Rohde, D. Kandhai, J. J. Derksen, and H. E. A. Van den Akker, Phys. Rev. E 67, 066703 (2003).

[21] S. Arcidiacono et al., Math. Comput. Simul. 72 (2-6), 79 (2006) 